\title{
Proactive interference in infant recognition memory
}

\author{
DONALD J. TYRRELL, LINDA G. SNOWMAN, JULIE A. BEIER, and CHRISTINE M. BLANCK \\ Franklin and Marshall College, Lancaster, Pennsylvania
}

\begin{abstract}
Preference for novel photographs of faces was tested in 5- to 7-month-old infants following three different proactive interfering conditions that replicated those in which retroactive interference had previously been demonstrated by Fagan (1973). One group of infants was presented with three successive presentations of upside-down caricatures of faces prior to the familiarization and novelty test, whereas the other two groups experienced three successive presentations of either upside-down or upright photographs of faces. Consistent with demonstrations of retroactive interference, preference for novelty existed following presentation of the rotated caricatures but not following rotated photographs. However, contrary to the effects due to retroaction, upright photographs did not lead to a preference for novelty. This differential disruption of preference for novelty is discussed as indicating either the existence of proactive interference in which the effects of perceptual similarity are different from those of retroaction, or the presence of a differential release from proactive interference that does not operate in retroaction.
\end{abstract}

Using the paired-comparison test of preference for novelty, Fagan $(1970,1973)$ demonstrated that infant recognition memory is quite robust, lasting over a period of 2 weeks following a 2-min exposure to a to-beremembered stimulus. Furthermore, he demonstrated several parallels between infant and adult memory phenomena, including differential forgetting due to retroactive interference and the elimination of interference effects if the presentation of the interfering event was delayed for $3 \mathrm{~h}$ following the initial recognition test-an effect consistent with the consolidation theory proposed by Hebb in 1949 .

Directly relevant to the current investigation was $\mathrm{Fa}$ gan's demonstration that delayed recognition of photographs of human faces by 21- to 25-week-old infants was disrupted by presentation of upside-down photographs of similar faces following the initial familiarization period. However, disruption was not created by upside-down line drawings of faces or by presentation of upright photographs of similar faces. He hypothesized that this pattern of differential disruption indicated interference related to the degree of perceptual similarity between the photographs employed in the test of preference for novelty and the interfering stimuli-with the line drawings being less

Partial support for the current research was provided by the Franklin and Marshall Committee on Grants and the Hackmann Scholar Summer Research Program administered by Franklin and Marshall. The authors extend their appreciation to Kenneth Ralph, Director of Information and Consultation for the Lancaster County Office of Mental Health and Mental Retardation, and to Donna Crosby, Director of the Lancaster County Mental Health Association, for their assistance in locating infants. Finally, appreciation is extended to all parents who volunteered their infants. Correspondence may be addressed to Donald J. Tyrrell, Department of Psychology, Franklin and Marshall College, P.O. Box 3003, Lancaster, PA 17604. similar than rotated photographs, and the upright photographs being highly similar. This interpretation is consistent with the Skaggs-Robinson hypothesis (Robinson, 1927) of interference in adult recall, in which interference varies as a function of stimulus similarity.

In the present experiment, we investigated differential disruption of memory in infants in a procedure analogous to that which produces proactive interference in adults (Underwood, 1957; Whitely, 1936). We reasoned that if memory in human infants is analogous to memory in older children and adults, then proactive interference effects should be demonstrable. Furthermore, if proaction and retroaction are affected by the similarity between the target and interfering information, similar patterns of disruption should occur. To test these possibilities, we presented infants with three 10-sec exposures to upsidedown line-drawn caricatures or upside-down and upright photographs of faces immediately prior to familiarization in a test of preference for novelty with upright photographs of faces. If, as Fagan reasoned, the rotated photographs are of an intermediate degree of perceptual similarity to the upright photographs, whereas the rotated caricatures are dissimilar, and if infant memory is subject to the effects of proaction in a manner analogous to the effects of retroaction, then interference should be created by the former, but not in the latter, condition. Finally, if the upright photographs are highly similar to the test photographs, they should not create interference.

In the paired-comparison paradigm, interference is indicated by a decrease in the preference for the novel stimulus. Thus, we predicted a significant preference for novelty following presentation of the rotated caricatures and the upright photographs, but a chance distribution of fixation following exposure to the rotated photographs. 


\section{METHOD}

\section{Subjects}

Thirty-five infants were tested. However, 3 ( 1 from each experimental condition) did not complete the experimental procedure due to fussiness. The remaining 32 infants, consisting of 15 girls and 17 boys ranging in age from 20 to 30 weeks (mean $=25$ weeks), were assigned to one of three experimental conditions. The infants presented with the upright photographs were tested only after testing of the infants in the other conditions was completed. However, there were no indications that these infants differed in any relevant respects (age, education, and occupation levels of the parents, length of gestation, etc.) from the infants in the first two groups.

\section{Stimuli and Apparatus}

Stimulus materials consisted of line-drawn caricatures of one male and two female human faces randomly selected from those described by Tyrrell, Anderson, Clubb, and Bradbury (1987) and the two sets of black-and-white photographs of male and female faces (M1, W1, and M2, W2) presented by Fagan (1973). All stimuli were mounted on white posterboard and affixed to wooden plaques that could be mounted on the apparatus by velcro strips, and were approximately $15 \mathrm{~cm}$ from chin to crown.

The stimuli were presented in an apparatus modeled after that described by Fagan (1970), the essentials of which included a portable testing chamber that contained a rotating stage upon which the stimuli were mounted. With the infant seated on a parent's lap and the stage in viewing position, the stimuli were approximately $35 \mathrm{~cm}$ from the infant's face. A small $(0.75-\mathrm{cm})$ peephole through which the infant's pupils could be observed was located midway between two $17 \times 17 \mathrm{~cm}$ plaques, which were affixed to the stage $35 \mathrm{~cm}$ apart (center to center).

\section{Design}

The experimental conditions consisted of three successive exposures to interfering stimuli followed by familiarization to one of the blackand-white photographs of a human face and an immediate preferencefor-novelty test during which the familiar and novel photographs were simultaneously presented.

One group of the infants was presented with successive exposures to the three line-drawn caricatures rotated $180^{\circ}$ from upright; infants in the second and third groups experienced three successive exposures to $180^{\circ}$ rotated or upright black-and-white photographs, respectively. Each exposure lasted until the infant accumulated $10 \mathrm{sec}$ of actual fixation to each stimulus, for a total of $30 \mathrm{sec}$ of interference.

The familiarization process consisted of two successive exposures to a single photograph with the left/right position changing over exposures. Each exposure continued until the infant accumulated $20 \mathrm{sec}$ of fixation to the photograph-thus, total familiarization time was $40 \mathrm{sec}$ of fixation. Preference for novelty was assessed during two successive periods during which the familiar and novel photographs were presented in adjacent positions, with the position changing between periods. Each period lasted for $10 \mathrm{sec}$ from the time of first fixation, and the total time each infant spent fixating each photograph during each period was recorded and summed across the two periods. The percentage of preference for novelty was calculated by dividing the total time the infant spent fixating the novel stimulus by the total fixation time to the two stimuli.

\section{Procedure}

All infants were tested in their homes at a time when the parents suggested they would be fed, rested, and most cooperative. After the procedure was explained to the parents and their permission to proceed was obtained, the infants were seated on the lap of a parent and the apparatus was positioned appropriately. For each exposure, the stimuli were mounted while the stage was rotated toward the experimenter out of the infant's sight. Then the stage was rotated toward the infant and the timing began. At the end of a period, a soft beep was emitted by the timer, the apparatus was rotated toward the experimenter, and the stimuli for the next period were mounted.

For the infants in the caricature condition, the three caricatures were presented in a random order with the positions counterbalanced over trials. For the photograph conditions, the two photographs from one set were presented in a random order over the three trials, and the photograph that was presented twice was counterbalanced over infants. The two sets of photographs served equally often as the interference and test sets, and the specific photographs in each set served equally frequently as the familiar and the novel stimulus.

\section{RESULTS}

The mean and standard deviations for the fixation times and the percent-novelty scores for the three conditions are presented in Table 1.

Both the fixation times and the percent-novelty scores were analyzed for any effects of sex of infant, and no main effects or interactions with sex were found. Therefore, for all remaining analyses, data were collapsed across sex.

A one-way analysis of variance on the percentage of total fixation time directed to the novel photograph indicated a significant main effect for experimental conditions $[F(2,29)=4.88, p<.05]$. Subsequent Newman-Keuls tests demonstrated that the only significant $(p<.05)$ difference was between the rotated photograph and rotated caricature conditions, whereas preference for novelty following the upright photographs was not different from either of the other conditions.

Separate $t$ tests assessing whether the percentage for each group differed from the chance expectation of $50 \%$ indicated a significant difference from chance in the caricature condition $[t(10)=3.87, p<.05]$, but in neither the rotated $[t(10)<1, p>.05]$ nor the upright photograph conditions $[t(9)=1.56, p>.05]$. Thus, there was no preference for fixating the novel stimulus in either of the photograph conditions, but in the rotated caricature condition the infants had a preference for the novel stimulus.

This pattern of novelty preferences is supported by a 3 (experimental conditions) $\times 2$ (novelty value) analysis of variance (with repeated measures on the latter factor) on fixation times. This analysis produced no main effect for condition $[F(2,29)=1.74, p>.05]$, but a significant effect for novelty versus familiarity $[F(1,29)=3.01$, $p<.05]$ and a significant interaction between the two $[F(2,29)=4.31, p<.05]$. Subsequent tests for simple main effects indicated a significant $(p<.05)$ effect for stimulus novelty in the rotated caricature condition but in neither of the other two conditions. Thus, the infants spent more time fixating the novel than the familiar stimu-

Table 1

Means and Standard Deviations of Fixation Times (in sec) to the Novel and Familiar Stimuli, and Percent-Novelty Scores (\% Novel) for Infants in All Experimental Conditions

\begin{tabular}{|c|c|c|c|c|c|c|}
\hline \multirow[b]{3}{*}{ Condition } & \multicolumn{4}{|c|}{ Fixation Time } & & \\
\hline & \multicolumn{2}{|c|}{ Novel } & \multicolumn{2}{|c|}{ Familiar } & \multicolumn{2}{|c|}{$\%$ Novel } \\
\hline & $M$ & $S D$ & $M$ & $S D$ & $M$ & $S D$ \\
\hline Rotate & 6.30 & 2.85 & 7.20 & 3.31 & 47.38 & 15.16 \\
\hline Rotated Caricatures & 8.45 & 1.92 & 4.95 & 1.85 & 63.26 & 11.34 \\
\hline Upright Photographs & 5.86 & 2.15 & 5.23 & 2.12 & 53.57 & 7.22 \\
\hline
\end{tabular}


lus in the rotated caricature condition, but not in the upright or the rotated photograph conditions.

\section{DISCUSSION}

Although the total time spent fixating the two stimuli during the novelty test was not affected by the specific stimulus set presented prior to the familiarization period, the infants presented with the rotated caricatures had a significant preference for the novel stimulus, whereas the infants presented with the photographs-regardless of their orientationdistributed their fixation time equally between the familiar and the novel stimulus.

Thus, we see a distinction between the processes associated with proaction and retroaction: when the interference conditions precede, rather than follow, the presentation of the to-be-remembered (i.e., familiar) stimulus, upright photographs interfere with performance on the novelty test in the same way that rotated photographs do-an effect that is not consistent with the results of Fagan (1973) with retroactive interference. Either the degree of perceptual similarity does not function in identical ways to produce retroactive and proactive interference, or some other process is operating to create interference during proaction.

One alternative process is the phenomenon of release from proactive interference (Wickens, 1970). This phenomenon has been demonstrated in elementary-school-age children with both verbal and pictoral stimuli (Tyrrell, Pressman, Cunningham, Steele, \& Thaller, 1981) and may have operated in the present experiment with infants.

If the rotated caricatures are encoded as instances of a different category of events than the upright photographs, response to the upright photographs for the infants presented with the caricatures would not be disrupted due to the change in encoding category and the resultant release from proactive interference. On the other hand, if the rotated and upright photographs are encoded as members of the same category, the response to the upright photographs during a test following earlier encoding of instances of the same category (both rotated and upright photographs) would be impaired through the continued presence of proactive interference.

In either case, it is possible that the infants were attending, encoding, and responding to some characteristic of the stimuli unrelated to their content. That is, the fact that there are faces represented in each of these presentation formats may be irrelevant to the infants. This is particularly likely, since the proportions of light and dark and, there- fore, overall brightness levels, differed between the line-drawn caricatures and the photographs, regardless of their orientation.

Finally, even though the effects of proaction did not duplicate the effects due to retroaction, the existence of, and possible release from, proactive interference provides additional support for the similarity in memorial function from infancy to adulthood. This similarity is crucial for the reasoning of those (e.g., Fagan \& Singer, 1983) who argue that the relationship between infant memory and later measured intelligence is due to the continuity in underlying processes throughout ontogeny.

\section{REFERENCES}

FAGAN, J. F., III (1970). Memory in the infant. Journal of Experimental Child Psychology, 9, 217-226.

FAGAN, J. F., III (1973). Infants' delayed recognition memory and forgetting. Journal of Experimental Child Psychology, 16, 424-450.

FAGAN, J. F., III, \& SINGER, L. T. (1983). Infant recognition memory as a measure of intelligence. In L. P. Lipsitt \& C. Rovee-Collier (Eds.), Advances in infancy research (Vol. 2, pp. 31-72). Norwood, NJ: Ablex.

HeBB, D. O. (1949). The organization of behavior. New York: Wiley. Robinson, E. S. (1927). The similarity factor in retroaction. American Journal of Psychology, 39, 297-312.

Tyrrell, D. J., Anderson, J. T., Clubb, M., \& Bradbury, A. (1987). Infant recognition of the correspondence between photographs and caricatures of human faces. Bulletin of the Psychonomic Society, 25, 41-43.

Tyrrell, D. J., Pressman, M., Cunningham, T., Steele, G. M., \& Thaller, K. (1981). Input and distractor modality effects upon the release from proactive interference in children. Journal of Genetic Psychology, 139, 205-220.

UNDERWOOD, B. J. (1957). Interference and forgetting. Psychological Review, 64, 49-60.

Whitely, P. L. (1936). The influence of certain conditions prior to learning upon subsequent recall. Journal of Experimental Psychology, 19, 496-504.

WICKENS, D. D. (1970). Encoding categories of words: An empirical approach to meaning. Psychological Review, 1, 1-15.

(Manuscript received September 25, 1989.) 